\title{
Effect of advanced platelet-rich fibrin applications on periodontal regeneration in infrabony pocket treatment
}

\author{
Christopher Imantaka Suwondo*, Dahlia Herawati**, Sudibyo** \\ *Dentes Dental Clinic, Pamecutan Klod, Denpasar, Bali, Indonesia \\ **Department of Periodontia, Faculty of Dentistry, Universitas Gadjah Mada, Yogyakarta, Indonesia \\ *JI Imam Bonjol 338A, Pemecutan Klod, Denpasar, Bali, Indonesia; email: christophersuwondo@gmail.com
}

Submitted: $1^{\text {st }}$ August 2018; Revised: 21 ${ }^{\text {st }}$ August 2018; Accepted: 28 ${ }^{\text {th }}$ November 2018

\begin{abstract}
One of the regenerative periodontal treatments for infrabony pocket is open flap debridement (OFD) with the addition of growth factor derived from platelet concentrate. Advanced platelet-rich fibrin (A-PRF) is a further development of plateletrich fibrin (PRF) with a lower centrifugation speed (1,500 rpm, 14 minutes). The purpose of this study was to examine the differences in periodontal tissue regeneration after the application of A-PRF and PRF in the treatment of infrabony pockets evaluated from probing depth (PD), relative attachment loss (RAL), and alveolar bone height. The samples were taken from 20 infrabony pockets divided into 2 groups: 10 subjects were treated with OFD+A-PRF and OFD+PRF on the remaining subjects. Probing depth (PD) and relative attachment loss (RAL) measurement were performed on days 0,30 , and 90. Bone height measurements were performed using CBCT X-rays on days 0 and 90 . The results showed that PD and RAL reduction in the group of OFD+A-PRF was significantly greater than that in the OFD+PRF group. Bone height reduction in both groups showed no difference. The conclusion obtained from this study is A-PRF application enhances periodontal tissue regeneration by generating greater probing depth and relative attachment loss reduction compared to $\mathrm{PRF}$, as well as an increase in bone height similar to in the treatment of infrabony pockets.
\end{abstract}

Keywords: advanced platelet-rich fibrin; infrabony pocket; open flap debridement; platelet-rich fibrin

\section{INTRODUCTION}

Periodontitis is an inflammatory disease of periodontal tissue that results in damage to periodontal ligament and alveolar bone. ${ }^{1}$ One of the clinical signs of periodontitis is the presence of a periodontal pocket. Periodontal pockets are divided into suprabony pocket and infrabony pocket. Infrabony pocket is a pocket with base of the sulcus more apical than alveolar bone, in general there is vertical or angular defect. ${ }^{2,3}$

Treatment of periodontitis includes surgical and non-surgical treatment. ${ }^{4}$ Surgical treatment is performed when inflammation persists after non-surgical treatment. ${ }^{5}$ One of the periodontal surgical treatments is an open flap debridement (OFD) aimed at removing focal infection, ${ }^{6}$ but could unable to regenerate tissue damaged by periodontal disease. ${ }^{7}$ The result of OFD treatment is the formation of long junctional epithelium. ${ }^{8}$
The primary goal of periodontal treatment is to regenerate periodontal tissue. ${ }^{9}$ Periodontal tissue regeneration is the formation of new bone, cementum, and new periodontal ligaments on tooth root surfaces that are damaged to restore anatomy and function. ${ }^{10}$ The clinical parameters for evaluating the success of periodontal regeneration are probing depth (PD), attachment loss, and bone height. ${ }^{8}$ Bone height can be seen through radiographic examination starting after 3 months. ${ }^{11}$

Ideal periodontal tissue regeneration is to improve periodontal and bone attachment, reduce pocket depth, and little gingival recession. ${ }^{12}$ Some types of regenerative periodontal treatment are: root conditioning, addition of bone graft, guided tissue regeneration (GTR), a combination of GTR-bone graft, and the addition of growth factor. ${ }^{13}$ Growth factors that contribute to periodontal regeneration include platelet-derived growth factor (PDGF), 
transforming growth factor (TGF- $\beta$ ), and insulin-like growth factor (IGF). ${ }^{14}$

The use of PRF in OFD treatment is to enhance tissue regeneration in the treatment of infrabony pocket. ${ }^{15}$ Platelet rich fibrin (PRF) is a second-generation platelet concentrate ${ }^{16}$ which is processed with whole blood taken from a patient fed into a $10 \mathrm{ml}$ tube and centrifuged immediately at 2,700 rpm, 12 mins, with PRF results in clot in the middle between acellular plasma and red blood cells. $17,18,19$

Platelet rich fibrin contains leukocytes, platelets, and growth factors contained in a threedimensional fibrin matrix that acts as a scaffold, ${ }^{20}$ thereby slowing the release of growth factors during wound healing. ${ }^{16}$ Some growth factors that can be found in PRF include PDGF, vascular endotel growth factor (VEGF), TGF- $\beta$, IGF-1, IGF-2, and epidermal growth factor (EGF). ${ }^{20,21}$

Some clinical applications of PRF are socket preservation after extraction, gingival recession treatment, infrabony defect regeneration, and sinus elevation. ${ }^{15}$ Treatment of infrabony defects by combining OFD and PRF gives better results than OFD alone. ${ }^{22}$ A study showed that the administration of demineralized freeze-Ddied bone allograft (DFDBA) as graft material in the treatment of infrabony pockets gave no significant difference with PRF. ${ }^{10}$

Advanced platelet-rich fibrin (A-PRF) is a further development of PRF by using the concept of lower rotational speed. A research on the effect of centrifugation forces on PRF protocol showed centrifugation pressure has an effect on cell distribution. ${ }^{23}$ Leukocytes in the PRF are mostly present at the bottom of the tube, due to centrifugation pressure. The procedure of preparing A-PRF by converting the centrifugation process to $1,500 \mathrm{rpm}$ for 14 minutes shows more evenly distributed granulocyte neutrophils ${ }^{16}$ and looser fibrin matrix. Neutrophil granulocytes have soft and hard tissue regeneration capabilities, direct monocytes to phagocytosis, and produce proteases such as MMP9 for wound healing process. ${ }^{23}$

A study comparing growth factor release in PRP, PRF, and A-PRF concluded that A-PRF released significantly more total factor growth than PRF. ${ }^{15} \mathrm{~A}$ research comparing PRF and A-PRF is limited to laboratory and animal studies, so further research with human subjects is needed.

\section{MATERIALS AND METHODS}

The type of research conducted was quasi experimental with independent variables OFD+A-PRF and OFD+PRF on the treatment of infrabony pocket with observation time of day 0 (baseline), day 30 , and day 90. Dependent variables were PD, RAL, and alveolar bone height.

After obtaining signed informed consent and ethical clearance (No.001220/KKEP/FKG-UGM/EC/2017), 20 infrabony pockets were divided into 2 groups, 10 subjects were treated with OFD+A-PRF and OFD+PRF on the other group. Periodontal depth and RAL measurements were performed on days 0,30 , and 90 . Bone height measurements were performed using CВCT X-rays on days 0 and 90.

Initial data collection of $\mathrm{PD}$ and RAL measurements was performed on the treated tooth. Probing depth was measured from the distance between gingival margin and sulcus, RAL was measured from cementoenamel junction (CEJ) and sulcus. CBCT X-ray was taken as initial data, then alveolar bone height was measured from the distance between CEJ to the most apical base of the defect from the vertical direction of the bone.

Periodontal flap surgical procedures began with local anesthesia, injected after the surgical area was disinfected. Full vertical and sulcular full thickness flap incisions were performed, flaps were observed and debridement was performed in the defect areas with SRP and curettage. Tetracycline $\mathrm{HCl} 75 \mathrm{mg} / \mathrm{ml}$ solution was applied to a hard tissue with sterile pellet cotton for 3 minutes which was changed every 30 seconds, and rinsed with distilled water.

Ten milliliter intravenous blood was taken and centrifuged according to their respective groups, A-PRF (1,500 rpm, 14 mins) and PRF (2,700 rpm, 12 mins). A-PRF and PRF clots were taken, partially clot was compressed on PRF box to membrane with its exudate called releasate. Clot, membrane, and releasate were applied to the infrabony defect. 
Majalah Kedokteran Gigi Indonesia. December 2018; 4(3): 154 - 160

ISSN 2460-0164 (print)

ISSN 2442-2576 (online)

The flap was returned and sutured, then covered with a periodontal pack. Patients were given antibiotics, analgesics and anti-inflammatory. The patients were given postoperative instructions and explanation how to maintain tooth and mouth health after surgery. Control was done 7 days later to remove the periodontal pack. Control of oral hygiene and wound healing was done once a week for 4 weeks after the OFD.

\section{RESULTS}

Table 1 shows that the mean of PD decreased on day 30 and day 90 (after treatment). The mean reduction of $P D$ after treatment (day 30 and 90) was greater in the OFD + A-PRF group than that in the OFD + PRF group. The initial values of PD measurement were different between the two treatment groups, so it is necessary to ascertain the magnitude of the reduction difference of PD in each group for further significance test.

The data in Table 2 show that the mean of PD reduction in the OFD + A-PRF group was greater than that in the OFD + PRF group on days 0-30, 30-90, and 0-90. The largest PD reduction was in the OFD + A-PRF group on days 0-90.

Mann-Whitney $U$ test of $P D$ reduction on days 30-90 showed no significant difference between the groups of OFD + PRF and OFD + A-PRF. Examination on days 0-30 and on days 0-90 showed a significance difference. Table 3 shows that PD reduction in A-PRF was significantly greater than that in PRF.

Table 4 shows that the mean of RAL decreased on day 30 and day 90 (after treatment). The mean of RAL reduction after treatment $\left(30^{\text {th }}\right.$ and $90^{\text {th }}$ day) was greater in the OFD + A-PRF group than that in the OFD + PRF group. The initial values of RAL measurements were different between the two treatment groups, so it is necessary to see the magnitude of the RAL reduction difference in each group for subsequent significance tests.

The data in Table 5 show that the mean of $\mathrm{RAL}$ reduction in the OFD + A-PRF group on days $0-30,30-90$, and $0-90$ was greater than that in the OFD + PRF group. The mean of RAL reduction on days 0-30 in both groups was greater than the mean of RAL reduction on 30-90 days. The mean of $R A L$ reduction was the highest on days 0-90 with mean and standard deviation of $3.40 \pm 1.26 \mathrm{~mm}$.

Mann Whitney $U$ test results on $R A L$ reduction on days 30-90 showed a significance value ofp $>0.05$ which means there was no significant difference in RAL reduction between OFD + PRF group and OFD + A-PRF group on days 30-90. The results on days $0-30$ and $0-90$ showed $p$ value $<0.05$. The table above shows that RAL reduction on A-PRF was greater than that of PRF significantly.

Table 7 shows alveolar bone height rate improved in the OFD + PRF group and the OFD + A-PRF group on day 90. Improved bone height was higher in the OFD + A-PRF group. Bone height improvement can be seen in Figure 1.

The significance level of parametric Paired T-test obtained $p$ value $<0.05$, indicating that there was significant alveolar bone height improvement between the observation time in each group. The initial values of alveolar bone height measurements between the two treatment groups were different. Therefore it was necessary to see the difference in alveolar bone height reduction in each group for subsequent significance tests.

Table 1. Mean and standard deviation of probing depth (PD) (mm)

\begin{tabular}{lccc}
\hline \multicolumn{1}{c}{ Time } & $\mathrm{n}$ & OFD + PRF & OFD + A-PRF \\
\hline Day-0 & 10 & $5.20 \pm 0.92$ & $5.10 \pm 0.99$ \\
Day-30 & 10 & $3.70 \pm 0.95$ & $3.00 \pm 1.15$ \\
Day-90 & 10 & $2.50 \pm 0.71$ & $1.30 \pm 0.48$ \\
\hline
\end{tabular}

Table 2. Mean and standard deviation of probing depth (PD) reduction $(\mathrm{mm})$

\begin{tabular}{cccc}
\hline Time & $\mathrm{n}$ & OFD + PRF & OFD + A-PRF \\
\hline Day 0 - 30 & 10 & $1.50 \pm 0.53$ & $2.10 \pm 0.57$ \\
Day $30-90$ & 10 & $1.20 \pm 0.92$ & $1.70 \pm 0.95$ \\
Day 0 - 90 & 10 & $2.70 \pm 0.95$ & $3.80 \pm 0.92$ \\
\hline
\end{tabular}

Table 3. Mann-Whitney $U$ test of probing depth (PD) reduction

\begin{tabular}{lc}
\hline \multicolumn{1}{c}{ Time } & $\mathrm{p}$ \\
\hline Day $0-30$ & $0.03^{*}$ \\
Day $30-90$ & 0.22 \\
Day $0-90$ & $0.02^{*}$ \\
\hline
\end{tabular}

*: significant 
Table 4. Mean and standard deviation of relative attachment loss (RAL) (mm)

\begin{tabular}{lccc}
\hline \multicolumn{1}{c}{ Time } & $\mathrm{n}$ & OFD + PRF & OFD + A-PRF \\
\hline Day-0 & 10 & $12.80 \pm 1.40$ & $12.10 \pm 1.10$ \\
Day-30 & 10 & $11.90 \pm 1.91$ & $10.30 \pm 1.42$ \\
Day-90 & 10 & $10.60 \pm 1.43$ & $8.70 \pm 1.06$ \\
\hline
\end{tabular}

Table 5. Mean and standard deviation of relative attachment loss (RAL) reduction

\begin{tabular}{cccc}
\hline Time & $\mathrm{n}$ & OFD + PRF & OFD + A-PRF \\
\hline Day $0-30$ & 10 & $0.90 \pm 0.74$ & $1.80 \pm 0.79$ \\
Day $30-90$ & 10 & $1.30 \pm 0.82$ & $1.60 \pm 1.51$ \\
Day $0-90$ & 10 & $2.20 \pm 0.79$ & $3.40 \pm 1.26$ \\
\hline
\end{tabular}

Table 6. Mann-Whitney $U$ test result of relative attachment loss (RAL) reduction

\begin{tabular}{lc}
\hline \multicolumn{1}{c}{ Observation time } & $p$ \\
\hline Day $0-30$ & $0.024^{*}$ \\
Day $30-90$ & 0.440 \\
Day $0-90$ & $0.040^{*}$ \\
\hline
\end{tabular}

*: significant

Table 7. Alveolar bone height mean and standard deviation ( $\mathrm{mm})$

\begin{tabular}{cccc}
\hline \multicolumn{1}{c}{ Time } & $\mathrm{n}$ & OFD + PRF & OFD + A-PRF \\
\hline Day-0 & 10 & $5.74 \pm 0.86$ & $5.08 \pm 0.74$ \\
Day-90 & 10 & $4.69 \pm 0.99$ & $3.77 \pm 0.77$ \\
\hline
\end{tabular}

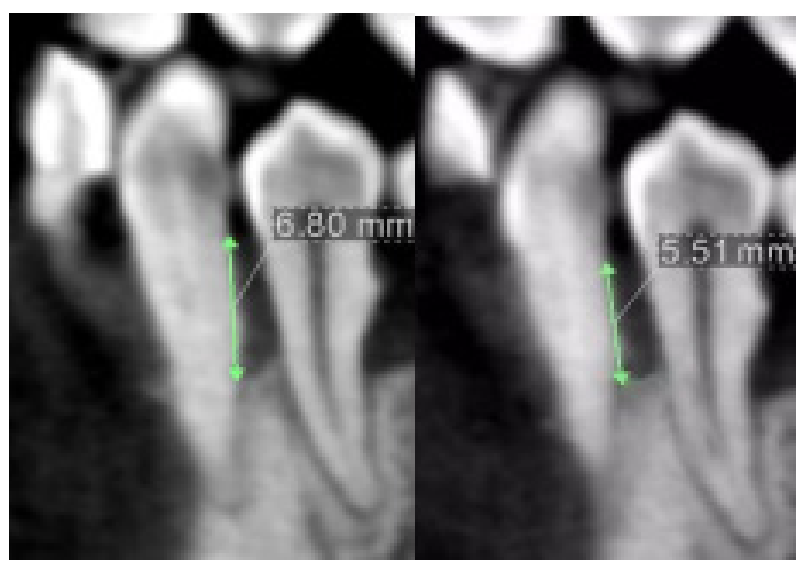

Figure 1. Alveolar bone height difference on day 0 and 90

Table 8. Parametric paired t-test results on alveolar bone height in each treatment group

\begin{tabular}{ccc}
\hline Group & $\mathrm{p}$ & \\
\hline OFD + PRF & 0.000 & Significant \\
OFD + A-PRF & 0.000 & Significant \\
\hline
\end{tabular}

Table 9. Mean and standard deviation of alveolar bone reduction $(\mathrm{mm})$

\begin{tabular}{cccc}
\hline Time & $\mathrm{n}$ & OFD + PRF & OFD + A-PRF \\
\hline Day $0-90$ & 10 & $1.04 \pm 0.49$ & $1.32 \pm 0.78$ \\
\hline
\end{tabular}

Table 10. Results of parametric independent t-test on alveolar bone height reduction between treatment groups

\begin{tabular}{cc}
\hline Observation time & $p$ \\
\hline Day $0-90$ & 0.355 \\
\hline
\end{tabular}

The results of independent t-test on alveolar bone height reduction showed a significance value of $p>0.05$, indicating there was no significant difference in alveolar bone height improvement between the OFD + PRF group and the OFD + A-PRF group.

\section{DISCUSSIONS}

The results showed $P D$ and RAL reduction in the OFD+A-PRF and OFD+PRF groups at each observation time, on day 30 and day 90 . This means there was an improvement in clinical conditions. Attachment loss is the distance between the base of the pocket with a CEJ or point and is generally related to probing depth, although it is not always the case. Reduced attachment loss values indicate a periodontal tissue repair. ${ }^{2}$

$P D$ and RAL reduction is influenced by growth factor produced by PRF and A-PRF, such as PDGF, EGF, and IGF-1 which act as homeostasis factor, increase proliferation and migration of periodontal fibroblasts, and prevent periodontal fibroblas apoptosis. ${ }^{1,24}$ The results of PD and RAL reduction between the two groups indicated a significantly greater PD and RAL reduction in the OFD+A-PRF group on days $0-30$ and days $0-90$. The reduction of the OFD+A-PRF group was significantly higher because the total number of growth factors produced by A-PRF was significantly higher than that of PRF. ${ }^{15}$ This allows for better tissue regeneration in both A-PRF groups. As is known, PDGF, VEGF, and IGF-1 increase the proliferation of fibroblasts and angioblasts, the synthesis of extracellular matrix, and angiogenesis process. ${ }^{25}$ More neutrophil granulocytes in A-PRF also affects macrophage differentiation, resulting in the regeneration of hard and soft tissues. ${ }^{16}$

The results of $P D$ and $R A L$ reduction between the OFD+A-PRF and OFD+PRF groups on days 30-90 did not show any significant differences. This is probably due to the fact that healing process 
enters tissue maturation process. As is known, the post-surgical treatment of periodontal surgery begins with an inflammatory process lasting up to two days postoperatively. On day 4, proliferation of fibroblasts and angioblasts occurs, followed by the formation of new junctional epithelium at the end of the 2 nd week. In the end of week 4 to month 3 , the process of tissue maturation and bone remodeling occurs. ${ }^{26}$

The results of alveolar bone height measurements obtained in this study between A-PRF and PRF applications was not significantly different. The median data showed an improvement in bone height on day 90 in both groups. This can be seen from the bone height on day 90 which was smaller than bone height on day-0 (baseline). The OFD+APRF group and the OFD+PRF group showed similar patterns of bone height improvement. Bone regeneration is affected by the association between RANK and RANKL or OPG. RANK binding to OPG may inhibit osteoclast activation and differentiation processes and increase osteoblast proliferation. ${ }^{2}$ The results suggest that $P R F$ and A-PRF may increase OPG secretion. ${ }^{27,28}$

Alveolar bone height reduction results showed a higher alveolar bone improvement in the OFD+APRF group compared to the OFD+PRF group. This is because in the process of A-PRF with lower centrifugation, the number of leukocytes obtained was higher because it did not sediment to the bottom of tube. ${ }^{23}$ Leukocytes play an important role in bone healing, especially macrophages. Macrophages improve the differentiation of osteoblasts that increase bone formation, and promote tissue integration. Leukocytes also prevent pathogen infiltration thereby reducing the risk of infection. ${ }^{16}$

The number of growth factors produced by A-PRF, such as PDGF, TGF, and VEGF, was significantly higher than that by PRF. ${ }^{15}$ VEGF plays an important role in the process of angiogenesis that stimulates the proliferation and migration of endothelial cells and maintains blood vessels formation. VEGF also plays a role in bone formation by increasing the migration, proliferation, and differentiation of osteoblasts. PDGF helps tissue regeneration and increases osteoblast precursor cells to stimulate bone regeneration. ${ }^{29}$ TGF increases the proliferation of mesenchymal cells, collects osteoblast precursor cells, and osteoblast differentiation. ${ }^{30}$

The alveolar bone height reduction difference between the OFD + PRF group and the OFD + A-PRF group showed no significant improvement in bone height, in line with a study of rabbit subjects. This can be due to several things, among others, PRF has a stronger structure than A-PRF when formed into membranes. The A-PRF membrane dissolves from day 3 , in contrast to the PRF membrane which can last up to 7 days. Total volume of PRF obtained after centrifugation is greater $\pm 30 \%$ than that of A-PRF. Based on histological examination, Bone Morphogenetic Protein (BMP)-2 was not found in the A-PRF group, whereas BMP-2 was found in the PRF group. ${ }^{28}$ Bone Morphogenetic Protein (BMP)-2 serves to improve osteoblast differentiation. In addition, the interaction between VEGF and BMP-2 may enhance the osteogenic effects of BMP-2. ${ }^{29}$

\section{CONCLUSION}

Based on the results of the study, it is concluded that periodontal tissue regeneration after APRF application seen from $P D$ and $R A L$ reduction is greater than that after PRF application on the treatment of infrabony pocket but there is no difference in alveolar bone height improvement.

\section{REFERENCES}

1. Mani R, Mahantesha S, Nandini TK, Lavanya R. Growth factors in periodontal regeneration. Journal of Advanced Oral Research. 2015; 5(2): 1-5.

2. Newman MG, Takei HH, Klokkevold PR. Carranza's Clinical Periodontology $11^{\text {th }}$ Edition. Missouri: Elsevier; 2012.

3. Bosshardt DD. The Periodontal pocket pathogenesis, histopathology, and consequences. Periodontology 2000. 2018; 76(1): 43-50.

4. Pradeep AR, Kanoriya D, Singhal S, Garg V, Guruprasad CN. Synergistic approach 
using platelet rich fibrin and 1\% alendronate for intrabony defect treatment in chronic periodontitis: a randomized clinical trial. Journal of Periodontology. 2016; 87(12): 1427-1435.

5. Heitz-Mayfield LJA, Lang NP. Surgical and nonsurgical periodontal therapy learned and unlearned concepts. Periodontology 2000. 2013; 62: 218-231.

6. Martande SS, Kumari M, Pradeep AR, Singh SP, Suke DK, Guruprasad CN. Platelet rich fibrin combined with $1.2 \%$ atorvastatin for treatment of intrabony defects in chronic periodontitis: a randomized controlled clinical trial. Journal of Periodontology. 2016; 87(9): 1039-1046.

7. Suchetha A, Lakshmi $P$, Bhat D, Mundinamane DB, Soorya KV, Bharwani GA. Platelet concentration in platelet concentrates and periodontal regeneration-unscrambling the ambiguity. Contemporary Clinical Dentistry. 2015; 6: 510-516.

8. Reynolds MA, Kao RT, Nares S, Camargo PM, Caton JG, Clem DS, Fiorellini JP, Geisinger ML, Mills MP, Nevins ML, Rosen PS. Periodontal Regeneration - Intrabony Defects: Practical Applications From the AAP Regeneration Workshop. Clinical Advances in Periodontics. 2015; 5(1): 21-29.

9. Hagi TT, Laugisch O, Ivanovic A, Sculean A. Regenerative periodontal therapy. Quintessence International. 2014; 45(3): 185-192.

10. Chadwick JK, Mills MP, Mealey BL. Clinical and radiographic evaluation of demineralized freeze-dried bone allograft versus platelet-rich fibrin for the treatment of periodontal intrabony defects in humans. Journal of Periodontology. 2016; 87(11): 1253-1260.

11. Crespi R, Cappare P, Gherlone E. MagnesiumEnriched hydroxyapatite compared to calcium sulfate in the healing of human extraction sockets: radiographic and histomorphometric evaluation at 3 months. Journal of Periodontology. 2009; 80(2): 210-218.
12. Cortellini P, Tonneti MS. Clinical concepts for regenerative therapy in intrabony defects. Periodontology 2000. 2015; 68: 282-307.

13. Illueca FMA, Vera PB, Cabanilles PG, Fernandez VF, Loscos FJG. Periodontal regeneration in clinical practice. Med Oral Patol Oral Cir Bucal. 2006; 11: 382-392.

14. Ivanovski S. Periodontal regeneration. Australian Dental Journal. 2009; 54(1): 118-128.

15. Kobayashi E, Fluckiger L, Fujioka-Kobayashi M, Sawada K, Sculean A, Schaller B, Miron RJ. Comparative release of growth factors from PRP, PRF, and Advanced-PRF. Clinical Oral Investigations. 2016; 20(9): 2353-2360.

16. Fujioka-Kobayashi M, Miron RJ, Hernandez M, Kandalam U, Zhang Y, Choukroun J. Optimized platelet rich fibrin with the low speed concept: growth factor release, biocompatibility and cellular response. Journal of Periodontology. 2017; 88(1): 112-121.

17. Ehrenfest DMD, Corso MD, Diss A, Mouhyi J, Charrier JB. Three-Dimensional architecture and cell composition of a choukroun's plateletrich fibrin clot and membrane. Journal of Periodontology. 2010; 81(4): 546-555.

18. Ehrenfest DMD, De Peppo GM, Doglioli P, Sammartino G. Slow release of growth factors and thrombospondin-1 in choukroun's plateletrich fibrin (PRF): a gold standard to achieve for all surgical platelet concentrates technologies. Growth Factors. 2009; 27(1): 63-69.

19. Toit JD, Gluckman H, Salama M. PlateletRich Fibrin (PRF): A Growth Factor-Rich Biomaterial. Part 1 - the Platelet Concentrates Milieu \& Review of the Literature. International Dentistry - African Edition. 2015; 5(5): 62-70.

20. Chandran P, Sivadas A. Platelet-Rich Fibrin: its role in periodontal regeneration. The Saudi Journal for Dental Research. 2014; 5: 117-122.

21. Christgau M, Moder D, Hiller KA, Dada A, Schimtz G, Schmalz G. Growth factors and cytokines in autologous platelet concentrate and their correlation to periodontal regeneration outcomes. Journal of Clinical Periodontology. 2006; 33: 837-845. 
22. Pradeep AR, Rao NS, Agarwal E, Bajaj P. Comparative evaluation of autologous platelet-rich fibrin and platelet-rich plasma in the treatment of three-wall inrabony defects in chronic periodontitis: a rancomized controlled clinical trial. Journal of Periodontology. 2012; 93(12): 1499-1507.

23. Ghanaati S, Booms P, Orlowska A, Kubesch A, Lorenz J, Rutkowski J, Landes C, Sader R, Kirkpatrick C, Choukroun J. Advanced Platelet-Rich Fibrin (A-PRF) - a new concept for cell-based tissue engineering by means of inflammatory cells. Journal of Oral Implantology. 2014; 40: 679-689.

24. Raja S, Byakod G, Pudakalkatti P. Growth factors in periodontal regeneration. International Journal of Dental Hygiene. 2009; 7: 82-89.

25. Pisoschi C, Stanciulescu C, Banita M. Growth factors and connective tissuehomeostasis in periodontal disease, pathogenesis and treatment of periodontitis. Prof. Nurcan Buduneli(Ed.); 2012. ISBN: 978-953-307-9240 . InTech,

Available:from:http://www.intechopen. com/books/pathogenesis-andtreatment-ofperiodontitis/growth-factors-and-connectivetissue-homeostasis-in-periodontal-disease
26. Prabhu $P$, Julius $A$, Elumalai $M$, Prabhu MN. Woung healing in periodontics. Biosciences Biotechnology Research Asia. 2014; 11(2): 791-795.

27. Chang YC, Wu KC, Zhao JH. Clinical application of platelet-rich fibrin as the sole grafting material in periodontal intrabony defects. Journal of Dental Sciences. 2011; 6: 181-188.

28. Titirinli K, Tekin U, Atil F, Onder E, Senguven B, Ozgul O, Kocyigit ID. Evaluation of advanced platelet rich fibrin (A-PRF) on Bone healing is it better than old version a histological animal study. Journal of Biomaterials and Tissue Engineering. 2017; 7(6): 478-483.

29. Yun YR, Jang JH, Jeon E, Kang W, Lee S, Won JE, Kim HW, Wall I. Administration of growth factors for bone regeneration. Regenerative Medicine. 2012; 7(3): 369-385.

30. Devescovi V, Leonardi E, Ciapetti G, Cenni E. Growth factors in bone repair. Chir Organi Mov. 2008; 92: 161-168. 